\title{
Obtenção On-line (https://covid-19.ufsj.tech) do Número de Leitos Municipais Demandados por COVID-19: popularização da ciência e apoio a políticas públicas
}

\author{
Obtaining Online (https://covid-19.ufsj.tech) the Number of Municipal \\ Beds Demanded by covid-19: science popularization and support for \\ public policies
}

\author{
Anderson Oliveira Latini ${ }^{1}$ \\ Celso Luiz Souza ${ }^{2}$ \\ Daniela Chaves Resende ${ }^{1}$ \\ Carla Fabiana Gouvêa Lopes ${ }^{2}$ \\ Dárlinton Barbosa Feres Carvalho ${ }^{1}$ \\ ${ }^{1}$ Universidade Federal de São João del-Rei, São João del-Rei, MG, Brasil \\ ${ }^{2}$ Instituto Federal-Sudeste, Campus São João del-Rei, São João del-Rei, MG, Brasil
}

\begin{abstract}
Resumo
Desde o final de 2019, a pandemia de COVID-19 tem demandado trabalho de cientistas em todo o mundo. Esta pesquisa usou o modelo matemático do Imperial College London para desenvolver um sistema de informações com dados demográficos dos 5.570 municípios brasileiros para a prospecção do número de leitos em diferentes cenários de isolamento, precoces e tardios, e publicação no site https://covid-19.ufsj.tech, permitindo o fácil acesso por pessoas comuns e por tomadores de decisão, com o intuito de auxiliar na popularização da ciência $e$ na produção de informações para o combate à pandemia de COVID-19. Em três meses de publicação do site, 2,6 mil usuários de ampla faixa etária e de 282 municípios fizeram 8,7 mil consultas pelo número de leitos em municípios. Em média, um usuário fez 3,4 consultas no tempo de 2,3 minutos. A informação disponibilizada e a facilidade de sua obtenção permitem classificar o site como ferramenta oportuna de divulgação de ciência e também de apoio a políticas públicas, já que cidadãos comuns e tomadores de decisão podem compreender melhor algumas das consequências de diferentes decisões acerca do isolamento social durante a pandemia.
\end{abstract}

Palavras-chave: SARS-CoV-2. Doença de Coronavírus. Brasil. Ferramenta on-line. Sistema Hospitalar.

\begin{abstract}
Since the beginning of 2020, the COVID-19 pandemic has mobilized scientists worldwide. This research used the Imperial College London mathematical model to develop an information system with demographic data from all Brazilian municipalities in order to inform the number of beds demanded in different isolation scenarios, early and late and publication in site https://covid-19.ufsj.tech permitting easily access by ordinary citizens and decision makers, helping in science popularization and information's production to deal with COVID-19 pandemic. After three months since site publication, 2,600 users of a wide age group and 282 municipalities made 8,700 consultations for the number of beds in published site. On average, users made 3.4 consultations in 2.3 minutes. The information available and the ease of obtaining it allow to classify the site as a timely tool for science's divulgation and also to public policies' support, since ordinary citizens and decision makers can better understand some of the consequences of different decisions about social isolation during COVID-19 pandemic.
\end{abstract}

Keywords: SARS-CoV-2. Coronavirus Disease. Brazil. Hospital System.

Área Tecnológica: Prospecção. Saúde. 


\section{Introdução}

A pandemia de COVID-19, que se iniciou na província de Wuhan na China (PEERI et al., 2020), apresenta uma porcentagem de fatalidade bastante similar à gripe espanhola e superior à Influenza A (H1N1) e à Influenza sazonal (SILVA, 2020). Em meados de maio de 2020, a pandemia já causava efeitos globais devastadores com o acúmulo de cerca de 5,5 milhões de casos registrados em todo o planeta, resultando em 350 mil mortes oficialmente ligadas ao vírus (WHO, 2020). Desse número de casos, aproximadamente 430 mil ocorrem no Brasil, com 26 mil óbitos registrados até 27 de maio de 2020. Apesar disso, segundo a Organização Mundial da Saúde (WHO, 2020), o pico de casos da pandemia ainda não foi registrado em muitos países, e o número de mortes provocadas pela COVID-19 continua a aumentar. O Brasil é um desses países em que o pico da pandemia ainda não foi alcançado e que possui a lamentável estimativa de que poderá alcançar mais de 125 mil mortos até o seu final, segundo aponta o Institute for Health Metrics and Evaluation (IHME, 2020a). Essas informações são alarmantes por si, mas, considerando vários indícios de subnotificação de casos e de mortes, então a questão faz-se incontestavelmente merecedora de grande atenção mundial.

Em resposta a circunstâncias como essas, pesquisadores de todo o mundo têm direcionado muito os seus esforços para a contenção do avanço da pandemia (TATUM et al., 2020). São muitas as frentes de pesquisa sobre a COVID-19, e, comumente, periódicos científicos têm dedicado fascículos inteiros para a divulgação dos avanços sobre o assunto. No Brasil, mesmo com a redução dos investimentos em ciência (ESCOBAR, 2015; 2019), a produção de trabalhos científicos sobre a COVID-19 disparou. Tais trabalhos têm focado, por exemplo, na qualidade das informações divulgadas (SOUSA-JÚNIOR et al., 2020), no efeito das políticas de quarentena no cenário atual e futuro (SARAIVA; OLIVEIRA; MOREJON, 2020), na prospecção e no desenvolvimento de testes clínicos e de fármacos para o tratamento da doença (QUINTELLA et al., 2020; OLIVEIRA, 2020) e nos impactos da pandemia sobre a economia (FERREIRAJÚNIOR; SANTA RITA, 2020), enfim, uma vasta gama de possibilidades em torno do vírus e sua consequente pandemia.

Dentro dos esforços científicos, a busca global por soluções, a mitigação da pandemia (e.g. LI et al., 2020; QUINTELLA et al., 2020; SILVA, 2020; VIGLIONE, 2020) e os estudos sobre a sua dispersão têm recebido atenção especial (KISSLER et al., 2020). Diversos modelos matemáticos foram e continuam a ser desenvolvidos para a obtenção de previsões sobre a doença, a sua dispersão e os efeitos de diferentes ações e intervenções que afetam o número de casos de infecção, o número de casos de infectados que desenvolvem os sintomas da doença e também o índice de infectados que necessitarão de leitos hospitalares e de unidades de terapia intensiva, as UTIs (MICHAUD; KATES; LEVITT, 2020; WALKER et al., 2020).

Na primeira semana de abril, uma revisão sistemática e crítica sobre esses modelos foi publicada no importante periódico científico, o British Medical Journal (WYNANTS et al., 2020), obtendo repercussão mundial. Nessa revisão, os autores, trabalhando com os modelos atuais preditivos para diagnósticos e prognósticos da infeção de COVID-19, confirmaram que esses modelos estão aparecendo rapidamente na literatura acadêmica como uma resposta da ciência ao necessário apoio às tomadas de decisão das nações. Contudo, os autores também apontam que os modelos propostos são comumente pouco detalhados, já que têm risco de vieses, e que ainda relatam desempenhos provavelmente otimistas do ponto de vista de sua operação. 
Desse modo, os autores fazem diversas considerações metodológicas a respeito da construção e da divulgação desses modelos, especialmente em relação à aderência que necessitam ter as diretrizes de relatórios do TRIPOD (relato transparente de um modelo de previsão multivariável para prognóstico ou diagnóstico individual).

Como isso se trata de simplificações de processos biológicos e sociais que se diferenciam em função de parâmetros (por exemplo, o distanciamento social adotado) e de procedimentos matemáticos utilizados, as projeções desses modelos matemáticos podem variar bastante (MICHAUD; KATES; LEVITT, 2020). O modelo matemático do IHME, um centro de pesquisas em saúde independente e sediado em Washington, previu cerca de 1.600 mortes devido à COVID-19 nos Estados Unidos para meados de abril, mas com grande variação, entre 362 e quase 5 mil mortes (IHME, 2020b). Da mesma forma, o IHME (2020c) projetou em 12 de março cerca de 90 mil mortes para o final de agosto próximo no Brasil e atualizou, 13 dias depois, para cerca de 125 mil mortes (IHME, 2020a).

Apesar dessa susceptibilidade inerente a modelos, o modelo publicado por Walker et al. (2020) tem recebido atenção especial, já que foi produzido especialmente para apoiar a tomada de decisões políticas e considera grande variedade de parâmetros e de previsões. Essa razão, somada ao modo de construção do modelo e à diversidade de cenários previstos, fez com que governos de diversos países tenham utilizado esse estudo em suas tomadas de decisão (ADAM, 2020).

Walker et al. (2020) simularam as consequências de diferentes estratégias que líderes de todas as nações do planeta poderiam tomar para o enfrentamento da pandemia de COVID-19. Entre essas estratégias, foram consideradas as seguintes: o não isolamento, o menor ou maior isolamento das populações (variando de 34 a 75\%), a propagação de infecções secundárias, o isolamento aprimorado para os mais idosos e os momentos de início de isolamento em função de número de mortos por habitantes. Os resultados obtidos neste estudo apontaram muitas relações de COVID-19 com, por exemplo, a proporção de idosos na população, o número de membros de diferentes idades nas famílias e o número de leitos hospitalares. Mas, destacam-se os dados relacionados à projeção de contágios, de número de casos com demanda hospitalar, de casos críticos e de mortalidade causada pela pandemia, sendo estes comparados entre cenários de não isolamento e de isolamento sob diferentes perspectivas. Os autores estimam que, caso nenhuma das nações do planeta interviesse utilizando o isolamento social rigoroso para o enfrentamento da pandemia, 7 bilhões de pessoas seriam infectadas pelo vírus e, dessas pessoas, cerca de 40 milhões morreriam em consequência direta da doença.

Os dados complementares do estudo de Walker et al. (2020) disponibilizam para todos os países do mundo, entre outras informações, os parâmetros que alimentaram os seus modelos, os resultados que obtiveram das suas simulações seguindo dois grandes cenários: a mitigação e a supressão de COVID-19. Sistematicamente, os resultados identificaram a impossibilidade de qualquer país do mundo conseguir absorver as demandas hospitalares da pandemia se a taxa de isolamento estiver entre 34 e $44 \%$, indicando a necessidade de isolamento mais eficaz. Além disso, também concluíram que a estratégia de supressão, correspondente ao isolamento social de pelo menos $75 \%$, precisa ser precocemente implementada para que se consiga reduzir consideravelmente a pressão sobre o sistema de saúde dos países e, consequentemente, o número de mortes, o que é válido para quaisquer dos países do planeta, sejam eles países ricos, países em desenvolvimento ou países pobres. 
O Brasil, país com 212,5 milhões de habitantes, ocupa, na data de 25 de junho de 2020, o segundo lugar no planeta em número de mortos registrados como COVID-19, com cerca de 54,4 mil mortes. Para lidar com o desafio imposto pela COVID-19, é fundamental o entendimento de muitas variáveis que influenciam a velocidade e o modo de sua dispersão, mas, também, é necessário conhecer a disponibilidade e a necessidade dos recursos para lidar com a situação, sendo especialmente importante a prospecção da demanda criada pela pandemia. Além disso, neste momento em que as informações para suporte da tomada de decisões são tão valiosas, é de fundamental importância a divulgação adequada de todas as informações com simplicidade e com qualidade visual, de modo a atingir não somente os tomadores de decisão, mas, também, a população comum. A partir dessas informações, tomadores de decisão têm mais fundamentação na avaliação de diferentes cenários para determinarem as suas ações, enquanto, por sua vez, a população comum (entenda-se por pessoas que não são políticos, ou médicos, ou cientistas) podem se apoderar de um conhecimento importante para cobrar por decisões mais acertadas a serem tomadas pelos políticos que as representam.

Considerando as circunstâncias da pandemia de COVID-19 e a necessidade de esforços científicos na direção de produção de ferramentas de apoio às decisões públicas, esta pesquisa pretende identificar as expectativas de total de infectados, o número total de leitos necessários e o número total de UTIs necessárias durante toda a pandemia e em seu pico, para todos os municípios brasileiros. Além disso, também se pretende disponibilizar um sistema de informação para divulgação on-line que permita a consulta dessas projeções por qualquer pessoa, seja ela político, cientista ou cidadão comum, para que possa acessar e formar melhor a sua opinião sobre o assunto, seja para tomar decisões ou para cobrar por elas.

\section{Metodologia}

Para possibilitar as análises obtidas no presente estudo, foram utilizados os resultados das estimativas do número de casos de infecção pela COVID-19 obtidos por Walker et al. (2020) para as diferentes nações do planeta. Entre esses resultados, os dados obtidos para o Brasil disponibilizados nos apêndices do estudo foram usados para balizar a interpretação e a construção dos dados para todos os 5.570 municípios brasileiros. Assim, as estimativas obtidas para o Brasil foram usadas para a construção de estimativas próprias dos diferentes municípios brasileiros utilizando, para cada um, o seu quantitativo populacional atualizado em janeiro do ano presente pelo Instituto Brasileiro de Geografia e Estatística (IBGE, 2020).

Posteriormente foi criado um banco de dados com informações demográficas de todos os municípios brasileiros, sendo este modelado por meio de uma engenharia reversa a partir dos dados disponibilizados pela plataforma do IBGE. Esse banco de dados foi validado pelo processo de normatização do modelo relacional, conforme mostra a Figura 1, com a finalidade de abrigar os dados que definem a população de todos os municípios do Brasil, além de armazenar as regras do modelo para possibilitar a prospecção das respostas para dois cenários de isolamento selecionados. Então, foi assumida a possibilidade da consulta por município, considerando-se o isolamento social de $75 \%$ da população mediante o gatilho de 0,2 mortos por semana por 100.000 habitantes (o primeiro gatilho, ou gatilho 1) ou então, quando mediante o gatilho de 
1,6 mortos por semana por 100.000 habitantes (o segundo gatilho, ou gatilho 2). A escolha desses dois cenários para a prospecção de consequências da pandemia para os municípios brasileiros advém do fato de que o nível de isolamento social desses dois cenários de supressão foi o necessário para produzir consequências absorvíveis pelo sistema de saúde das nações do mundo, sejam estas nações ricas, nações em desenvolvimento ou nações pobres.

O banco de dados foi populado por todos os dados de identificação e de população de cada um dos municípios brasileiros (IBGE, 2020). Além disso, também foram ajustados os fatores matemáticos que permitiram gerar os dois cenários distintos: o início do isolamento de forma precoce (gatilho 1) ou de forma tardia (gatilho 2) para cada consulta por município. Para a divulgação dos dados obtidos na busca, foi construído um website com interface descrita em HTML (Hypertext Markup Language) e implementação das funcionalidades por meio da linguagem de programação PHP (Hypertext Preprocessor), sendo desenvolvido um mecanismo de recuperação de informações do banco de dados criado, apresentando os cenários distintos com a finalidade de publicar um site na internet.

O site publicado na internet, para acesso de qualquer pessoa, foi hospedado no endereço eletrônico https://covid-19.ufsj.tech/. O mecanismo de busca foi posicionado na página de abertura do site, com o propósito de torná-lo mais prático e objetivo em sua função. Em páginas secundárias foram ainda disponibilizadas informações sobre as principais referências bibliográficas utilizadas, sobre os autores e também sobre as instituições envolvidas, além de um texto explicativo da motivação da criação do site. O site foi desenvolvido com design responsivo, permitindo que fossem visualizadas e mantivessem suas funcionalidades por uma variedade de dispositivos com telas ou janelas de diferentes tamanhos, como desktop, celulares e tablets. Para aumentar as funcionalidades do site, também foi inserido um link a um site secundário que analisa o nível de isolamento da sociedade brasileira, podendo este ser acompanhado ao longo do tempo para o país inteiro ou para Estados específicos (https://www.inloco.com.br/).

Figura 1 - Ilustração do banco de dados para a prospecção do número de leitos, número de UTIs e número de UTIs no pico da pandemia para todos os municípios brasileiros

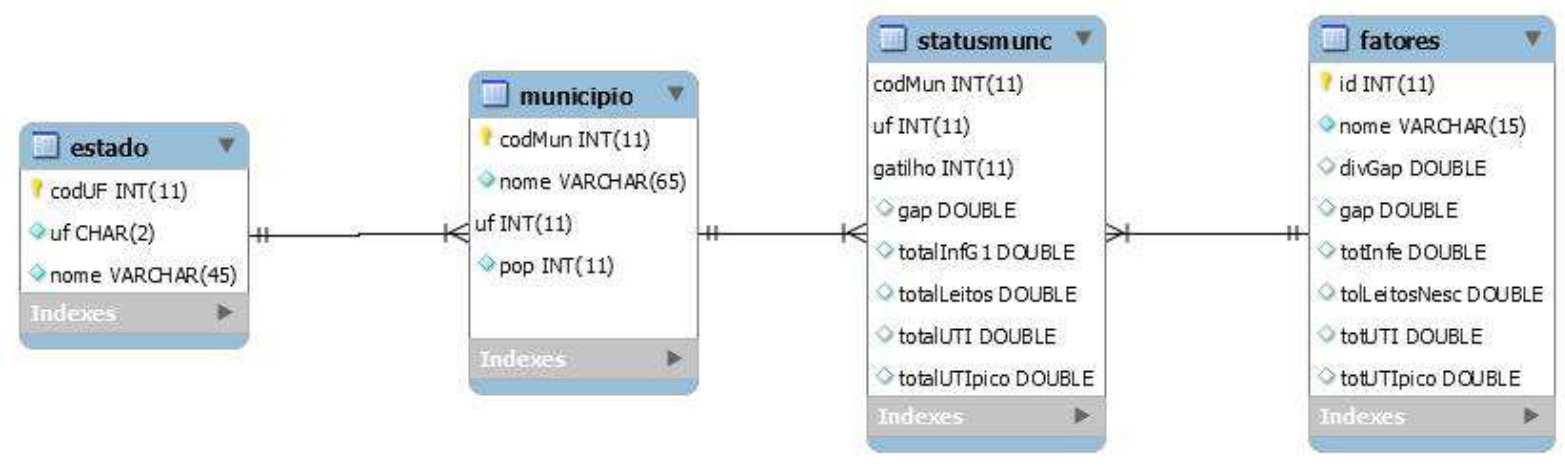

Fonte: Elaborada pelos autores deste artigo (2020)

Após a publicação do site, foi utilizado o Google Analytics para a monitoração do uso do site. Essa é uma ferramenta gratuita de monitoramento e de análise de sites que permite analisar o uso do website para aplicações diversas. Para isso, ela coleta dados dos usuários, permite um gerenciamento personalizado de como esses dados serão processados, realiza o processamento e fornece 
acesso aos dados obtidos. Com o seu uso, foi possível obter o número de visualizações, o número de usuários, as páginas mais acessadas dentro do site, os dispositivos utilizados para acessá-la (celular, desktop ou tablets), país e cidade de acesso e o modo como acessaram a página (link direto, link indicado por outra página ou mídia social), permitindo uma boa análise inicial do uso do site.

\section{Resultados e Discussão}

Após a publicação do banco de dados (https://covid-19.ufsj.tech/) com os 5.570 municípios brasileiros, foi possível obter um estudo analítico de seu uso e uma interpretação sobre sua aplicabilidade ao fomento de políticas públicas, como descrito a seguir.

\subsection{Prospecção de Leitos Demandados para os Municípios do Brasil}

As informações produzidas para o Brasil, em diferentes cenários, encontram-se dispersas em planilhas de anexos do trabalho de Walker et al. (2020), sendo elas de difícil entendimento para uma pessoa não habituada à leitura e à interpretação de resultados científicos. A organização e a tabulação desses dados tornaram possível sua melhor interpretação para o Brasil, além da geração de novos dados para o seu uso em estimativas para os municípios brasileiros.

Em nível de país, é inevitável a estratégia de supressão da pandemia com $75 \%$ de isolamento social no Brasil, já que, independentemente do momento em que tal decisão for tomada, sendo o isolamento precoce (com 0,2 mortos por 100 mil habitantes) ou tardio (com 1,6 mortos por 100 mil habitantes), a demanda de leitos deve cair praticamente 30 vezes em relação à decisão de não isolamento (diferença encontrada entre a decisão de isolamento no gatilho $1 \mathrm{em}$ comparação à decisão de não isolamento).

O uso de isolamento social de forma tardia pode levar a um aumento de cerca de $470 \%$ do número de pessoas contaminadas, hospitalizadas, hospitalizadas em estado crítico e também do número de pessoas mortas pela COVID-19. É importante salientar que a diferença na demanda de leitos prevista é de 460.361, e a demanda de UTIs é de 97.044. Por outro lado, caso o distanciamento social intensivo se inicie no primeiro gatilho, haveria a demanda estimada de 72.398 leitos e de 15.432 UTIs no pico, ou seja, um número seis vezes menor de leitos que a demanda estimada quando o isolamento é acionado no segundo gatilho, o que muda completamente o cenário de enfrentamento da pandemia no Brasil.

Traduzindo para as potencialidades nacionais, em abril, o Brasil declarava possuir cerca de 55 mil leitos de UTI (MINISTÉRIO DA SAÚDE, 2020). A adoção do cenário de supressão, considerando isolamento social intensivo, com cerca de $75 \%$ da população, quando ocorrerem 0,2 mortes por semana por 100 mil habitantes (primeiro gatilho), prevê um cenário no qual o Sistema de Saúde brasileiro tem condições de absorver os impactos da COVID-19. Essa é uma boa notícia, mas deve estar acompanhada da condição de existência de distanciamento social intensivo disparado de forma precoce. 
Os parâmetros utilizados no modelo para o Brasil refletem em seus municípios, já que estes contribuem com informações específicas que auxiliam na informação generalizada do país. Assim, as conclusões do estudo em escala nacional foram utilizadas para a obtenção de dados que refletem conclusões possíveis para os municípios brasileiros permitindo que se possa saber qual seria a resposta esperada por município, utilizando as estimativas obtidas no estudo para a supressão nos dois diferentes gatilhos.

Após a construção do banco de dados para os municípios brasileiros, um website foi disponibilizado na internet, sendo acessível no endereço http://covid-19.ufsj.tech/ que fornece a resposta dessa prospecção para qualquer município do país (SOUZA et al., 2020). Por meio de consulta a essa página, é possível obter informações relacionadas às consequências da pandemia sobre os sistemas de saúde municipais, como o caso ilustrado na Figura 2.

Para exemplificar, será tomado como exemplo o município de Belo Horizonte, capital do Estado de Minas Gerais, que foi utilizado para a elaboração da Figura 2. Tal município tem uma população estimada em cerca de 2,5 milhões de habitantes, segundo o IBGE (2020), e o primeiro gatilho da ação de supressão social com isolamento de $75 \%$ da população, corresponde ao registro de cinco mortes pela COVID-19 por semana. Já o segundo gatilho corresponde a 40 mortes por semana, aplicando-se em ambos os gatilhos as condições preconizadas pelo modelo. Além da grande diferença no número de mortos, há uma grande diferença na demanda por UTIs no pico da pandemia na cidade, sendo que, com a supressão acionada no primeiro gatilho, 181 UTIs seriam necessárias, enquanto, caso o acionamento do isolamento por supressão seja feito no segundo gatilho, a demanda muda para 1.130 UTIs, um número seis vezes superior.

É possível apontar como um novo exemplo para a cidade de São Paulo, considerando-se a população estimada em cerca de 12,2 milhões de habitantes, segundo o IBGE (2020), os gatilhos 1 e 2 correspondem a 24 e 196 mortes pela COVID-19 por semana. A demanda por UTIs nessa cidade, no pico da pandemia, tem a projeção de 886 no primeiro gatilho e a projeção de 5.219 no segundo gatilho, um número praticamente seis vezes superior.

Os resultados obtidos, tanto para Belo Horizonte como para São Paulo, são representativos das consequências do uso de um isolamento social tardio nas cidades brasileiras. Mas, por outro lado, os números obtidos aliados às notícias das ações que ambos os municípios tomaram, de isolamento social intensivo precoce e anterior ao número de mortes do segundo gatilho, indicam que os dois municípios têm boa possibilidade de absorver os impactos diretos da COVID-19 em seus sistemas de saúde, em especial porque também têm tomado ações emergenciais.

A transposição das informações produzidas pelo trabalho do Imperial College London para a prospecção das consequências, em nível de municípios brasileiros, permite uma projeção simulada dos efeitos das ações pelo não isolamento ou pelo isolamento social intensivo, mediante os gatilhos de 0,2 ou 1,6 mortos por 100.000 habitantes por semana para todos os municípios brasileiros. Assim, cada gestor tem disponível uma boa ferramenta para ajustar a realidade de seu município e tomar a sua decisão, caso possa ser distinta de uma decisão estadual ou federal. 
Figura 2 - Ilustração de um resultado obtido da busca feita no site http://covid-19.ufsj.tech/ (SOUZA et al., 2020) para o município de Belo Horizonte, MG

\begin{tabular}{|c|c|c|c|}
\hline \multicolumn{2}{|l|}{ Gatilho 1 A } & \multicolumn{2}{|l|}{ Gatilho 2 B } \\
\hline & Número & & Número \\
\hline Gatilho & 5,02 & Gatilho & 40,19 \\
\hline Total de infectados & 133.140 & Total de infectados & 577.776 \\
\hline Total de leitos necessários & 52.753 & Total de leitos necessários & 57.778 \\
\hline $\begin{array}{l}\text { Total de UTIs necessárias (todo período da } \\
\text { epidemia) }\end{array}$ & 678 & $\begin{array}{l}\text { Total de UTIs necessárias (todo período da } \\
\text { epidemia) }\end{array}$ & 3.014 \\
\hline Total de UTIs no pico da pandemia & 181 & Total de UTIs no pico da pandemia & 1.130 \\
\hline \multicolumn{2}{|c|}{$\begin{array}{l}\text { A Estas são as previsões do modelo de Walker e } \\
\text { colaboradores (2020) para o caso do seu município tomar a } \\
\text { decisão de isolamento social quando alcançar 0,2 mortos } \\
\text { por Covid- } 19 \text { para cada } 100.000 \text { habitantes por semana e } \\
\text { alcançar ao menos } 75 \% \text { de isolamento. }\end{array}$} & \multicolumn{2}{|c|}{$\begin{array}{l}\text { B Estas são as previsões do modelo de Walker e } \\
\text { colaboradores (2020) para o caso do seu município tomar a } \\
\text { decisão de isolamento social quando alcançar } 1,6 \text { mortos } \\
\text { por Covid-19 para cada } 100.000 \text { habitantes por semana e } \\
\text { alcançar ao menos } 75 \% \text { de isolamento. }\end{array}$} \\
\hline
\end{tabular}

Fonte: Elaborada pelos autores deste artigo (2020)

Do mesmo modo, cada habitante tem acesso a tais dados e pode exercer o seu direito de requisitar ações específicas do poder público, uma vez que conhece as consequências simuladas para o seu município. Se cada município tem realidades econômicas e sociais diferentes, então, tais informações também podem auxiliar na organização de uma estrutura para busca e oferta de leitos excedentes em outros municípios, otimizando a capacidade de lidar com a pandemia no Brasil.

\subsection{Estudo Analítico do Uso do Site}

Após a sua construção, a divulgação do site foi feita via redes sociais, em um jornal on-line e nos sites do Instituto Federal-Sudeste de Minas Gerais e da Universidade Federal de São João del-Rei. Segundo a análise fornecida pelo Google Analytics, em 90 dias de disponibilidade do site (http://covid-19.ufsj.tech), foram feitas 8.700 consultas (média de 97 consultas diárias) ao número de leitos necessários para absorver o impacto da pandemia de COVID-19. Essas consultas foram feitas por 2.496 usuários diferentes, significando uma média de 3,5 consultas por usuário. Cerca de $85 \%$ desses usuários fizeram uma visita e não retornaram mais ao site, pelo menos não com o mesmo dispositivo.

Os acessos ao site foram feitos a partir de 282 municípios de todo o planeta, com aproximadamente 93\% dos acessos (8.071) feitos do Brasil, 4,5\% dos Estados Unidos (a maioria do Estado do Kansas) e os restantes 2,5\% de outros 23 países. Em relação aos usuários brasileiros, $74 \%$ são do Estado de Minas Gerais, e o município de Belo Horizonte contribuiu com a maior parte dos acessos nacionais, 13,5\%. Apesar dos acessos virem de 282 municípios, a décima 
cidade com maior percentual de acessos, Brasília, deteve 2,1\% dos acessos, indicando uma boa distribuição dos acessos entre os diferentes municípios.

Cerca de $75 \%$ dos acessos foram feitos por aparelhos de telefonia celular, $23 \%$ por meio de desktops e $2 \%$ a partir de outros dispositivos, como tablets. O tempo médio despendido por usuário em seus acessos foi de 2 minutos e 36 segundos, indicando a capacidade do site em fornecer a informação desejada pelos usuários de forma rápida e objetiva. Essa indicação é ainda corroborada pelo fato de que $89 \%$ dos usuários acessaram somente a página inicial do site, onde se localiza o mecanismo de busca municipal pelo número de leitos demandados durante a pandemia.

O Google Analytics permitiu identificar ainda que 47,7\% dos acessos foram feitos por pessoas do gênero feminino e 52,3\% foram acessados do gênero masculino. Cerca de $19 \%$ desses usuários tinham menos de 24 anos, 35\% entre 25 e 34, 17\% entre 35 e 44 e cerca de $28 \%$ igual ou acima de 45 anos de idade.

A divulgação de ciência e tecnologia é determinante para o desenvolvimento de qualquer sociedade e pode afetar os seus valores e suas atitudes, seu bem-estar e segurança e até mesmo a sua própria existência. Professores e pesquisadores vêm usando novas formas lúdicas em sala de aula (e.g. FACCIONI; SOLER, 2018), estudando e testando ferramentas inusitadas, como a literatura de cordel (ALMEIDA et al., 2016) e histórias em quadrinhos (EVANGELISTA, 2018) com o intuito de contribuírem para uma melhor educação e para a construção de valores da sociedade, contexto em que o teatro vem ganhando atenção especial para o ensino e a divulgação da ciência (ALMEIDA et al., 2018; MOREIRA, 2015; PEREIRA, 2018).

Desse modo, o site desenvolvido nesta pesquisa também se apresenta como ferramenta alternativa para a divulgação e a popularização da ciência. A interpretação de dados de um modelo matemático construído para todos os países do mundo e publicado em outra língua (WALKER et al., 2020) e a sua transposição para os municípios brasileiros com a possibilidade de fácil consulta por meio de uma ferramenta de acesso universal auxilia na divulgação da ciência. A quantidade de consultas ao site, a diversidade de municípios de origem dos usuários, o curto tempo despendido na pesquisa e a equidade de faixas etárias dos usuários ilustram que o site se apresenta como ferramenta eficaz na divulgação de conhecimento científico e, especificamente, na divulgação de conhecimento relevante em relação à pandemia de COVID-19. Além disso, o seu uso por tomadores de decisão também tem potencial para apoiar políticas públicas.

\subsection{Aplicabilidade do Site para Políticas Públicas}

Mas, qual a fidelidade e qual crédito os resultados apresentados pelo site podem ter? Modelos são usados para prever diferentes fenômenos no mundo, entre eles, econômicos (HIMICS; LISTORTI; TONINI, 2020), meteorológicos (HEAVENS; WARD; MAHOWALD, 2013), ambientais (HARAMBOURE et al., 2020) e populacionais, como é o caso dos modelos para projeção de consequências das decisões em função da pandemia de COVID-19 (MICHAUD; KATES; LEVITT, 2020; WYNANTS et al., 2020; WALKER et al., 2020). Modelos são construídos para projetar cenários e, mesmo quando trazem menos acertos, eles têm grande importância para a análise e a tomada de decisões, inclusive em relação a medidas referentes à saúde pública mundial, como é o caso que a humanidade vive agora (ADAM, 2020; MICHAUD; KATES; LEVITT, 2020). 
Considerando as consequências da COVID-19 sobre os sistemas de saúde dos diferentes municípios brasileiros e o entendimento e a tomada de decisão de políticos acerca do isolamento social, pode-se imaginar quatro combinações possíveis de fatos:

a) A primeira é: se a decisão pelo isolamento social intensivo, ou seja, ao menos $75 \%$ da população, for tomada e a COVID-19 não tiver o mesmo poder nos municípios brasileiros que apresentou em municípios de outros países, então a decisão pelo isolamento seria somente preventiva e a consequência seria inócua sobre a propagação do vírus. Tal possibilidade já se encontra afastada, já que o número de mortes que o país acumula em meados de maio, cerca de 26 mil (WORLDOMETERS.INFO, 2020), ilustra que o vírus tem potencial letal devastador no Brasil, assim como em outros países.

b) A segunda combinação advém da decisão do isolamento social intensivo e, ao mesmo tempo, o vírus possuir o potencial devastador que demonstra no exterior. Nesse caso, então, a decisão de isolamento é acertada e poderá prevenir milhares de mortes no Brasil. Nos Estados Unidos, por exemplo, o doutor Anthony Fauci sugeriu que muitas vidas poderiam ter sido salvas, caso o isolamento tivesse se iniciado mais cedo e em meados de maio, o país amarga cerca de 100 mil mortos.

c) A terceira combinação agregaria a decisão de não isolamento social intensivo e a manifestação do vírus com o mesmo potencial devastador que demonstra no exterior, fato já conhecido como real. Nesse caso, o erro da decisão pública seria muito grave e os municípios brasileiros poderiam vivenciar uma taxa de mortalidade catastrófica.

d) A última combinação seria obtida não sendo realizado o isolamento social intensivo e não havendo, no Brasil, o potencial devastador que a COVID-19 demonstra no exterior. Nesse caso, o governo (municipal, estadual ou federal) assumiria um risco muito elevado, mas estaria sendo blindado pela sorte, não colhendo os piores resultados possíveis. Baseado em dados científicos e nos casos já em curso pelo mundo, não somente em modelos matemáticos, uma decisão como essa se enquadraria em uma gestão de alto risco. Contudo, o número de mortes já registrado no país ilustra que essa combinação não tem chances de ocorrer.

O uso de modelos para projeções de uso de leitos ou de leitos de UTIs e para a projeção de fatalidades joga luz sobre informações importantes para gestores e cidadãos brasileiros $e$ possibilita a melhor compreensão das consequências de diferentes decisões que podem ser tomadas em relação ao isolamento da população. Dados obtidos em meados de abril por localização embarcada em celulares (https://content.inloco.com.br/) ilustram que a população estava disposta ao isolamento, alcançando cerca de $60 \%$ de efetivo isolamento, ou seja, muito próximo da taxa sugerida de supressão pelo modelo de Walker et al. (2020). Contudo, os dados atuais da mesma fonte ilustram o relaxamento desse isolamento, o que constitui grande risco, considerando que o pico da pandemia ainda não foi atingido. 


\section{Considerações Finais}

Isolamentos sociais brandos ou a inexistência deles são capazes de causar uma tragédia no sistema de saúde brasileiro. Por outro lado, o isolamento social de pelo menos $75 \%$ da população gera consequências mais prováveis de serem absorvidas pelo sistema de saúde nacional. Essas informações foram simplificadas visualmente neste trabalho e disponibilizadas on-line no site https://covid-19.ufsj.tech/ para a consulta de cada município brasileiro, permitindo seu conhecimento por qualquer cidadão, incluindo aqueles que executam o papel de tomadores de decisão. O número e origem dos usuários, considerando os primeiros 90 dias de uso, assim como o número de consultas que fizeram, ilustram que o site tem potencial para divulgar e popularizar um conhecimento científico de relativa complexidade para ser obtido, já que foi realizado por meio de modelos matemáticos, que consistem em representações da realidade baseadas em diversas variáveis e parâmetros. Considerando o número de acessos e sua origem, então é evidente que pessoas comuns se apoderaram desse conhecimento, o que permite que elas somem informações para exercerem o direito de cobrar pelas melhores escolhas feitas pelos tomadores de decisão. Do mesmo modo, o número de usuários e a sua origem também permitem concluir que o site publicado e o seu conhecimento associado alcançaram, pessoalmente ou profissionalmente, governantes ou seus representantes, dando a oportunidade de se apoiarem nessa ferramenta para tomarem melhores decisões em relação ao isolamento social.

\section{Perspectivas Futuras}

A atual pandemia de COVID-19 demanda para o seu entendimento e enfrentamento esforços de cientistas de todas as áreas. São notoriamente importantes os esforços de cientistas da área da saúde e de áreas correlatas, sobretudo a respeito da transmissão e do tratamento da doença, da divulgação de apontamentos diversos sobre a dispersão do vírus e de fatores que mitiguem a pandemia. Contudo, também são notáveis os esforços de cientistas de outras áreas que têm retornado benefícios a seu enfrentamento, por exemplo, na projeção de consequências diversas do isolamento sobre a sociedade global e sobre diferentes faixas etárias, além de consequências econômicas.

Tal mobilização da comunidade científica em busca de entendimento detalhado acerca da pandemia de COVID-19 não tem precedentes, assim como os esforços para a popularização de conhecimentos para toda a sociedade. A popularização de tais conhecimentos além de ser relevante é uma tendência neste momento de pandemia, já que a capacitação das pessoas a respeito da pandemia eleva a sua habilidade em exigir por seus direitos e por respostas adequadas do poder público.

Um exemplo disso são os resultados que esta pesquisa apresenta, além da simplificação da visualização de resultados projetados para o Brasil, aqui foi apresentado um banco de dados de acesso universal com a prospecção de demanda hospitalar para cada município brasileiro durante toda a pandemia. Desse modo, o trabalho e o site disponibilizado constituem mais uma informação auxiliar para a sociedade no combate à COVID-19, já que conhecer a prospecção dessa demanda de leitos é fundamental para que os setores de saúde municipais possam se posicionar melhor em relação às decisões de suas administrações públicas. O melhor entendi- 
mento dessa demanda de leitos permite auxiliar em como se dará o fluxo de pacientes e quais são recursos do sistema de saúde de cada município que estão sobrando ou faltando para lidar com a pandemia, além disso, permite que pessoas comuns possam interpretar as consequências das decisões políticas sobre o isolamento social e possam exercer o direito de cobrar por decisões mais acertadas dos seus representantes.

\section{Agradecimentos}

Agradecemos a um colega da área médica que está lidando diariamente com problemas causados pela COVID-19 e que nos deu maior compreensão das importantes demandas para lidar com o problema. Esse colega preferiu não ser identificado neste trabalho.

\section{Referências}

ADAM, D. Special report: The simulations driving the world's response to COVID-19. Nature, [S.l.], v. 580, p. 316-318, 2020. DOI: 10.1038/d41586-020-01003-6.

ALMEIDA, C. S. et al. Ciência e teatro : um estudo sobre as artes cênicas como estratégia de educação e divulgação da ciência em museus. Ciência e Educação, [S.l.], v. 24, n. 2, p. 375-393, 2018.

ALMEIDA, C.; MASSARANI, L.; MOREIRA, I. DE C. Representações da ciência e da tecnologia na literatura de cordel. Bakhtiniana: Revista de Estudos do Discurso, [S.I.], v. 11, n. 3, p. 5-25, 2016.

ESCOBAR, H. Fiscal crisis has Brazilian scientists scrambling. Science, [S.l.], v. 349, n. 6.251, p. 909-910, 2015. DOI: 10.1126/science.349.6251.909.

ESCOBAR, H. Brazilian scientists lament "freeze" on research budget. Science, [S.l.], v. 364, n. 6.436, p. 111, 2019. DOI: 10.1126/science.364.6436.111.

EVANGElistA, T. S. Relato de experiência. Caminho Aberto - Revista de Extensão do IFSC, [S.l.], v. 5, n. 8, p. 83-88, 2018.

FACCIONI, L. C.; SOLER, R. R. Abordagem lúdica sobre os aspectos celulares do diabetes e da obesidade para alunos do ensino médio. Revista Brasileira de Extensão Universitária, [S.l.], v. 9, n. 1, p. 27-37, 2018.

FERREIRA-JÚNIOR, R. R.; SANTA RITA, L. P. Impactos da Covid-19 na Economia: limites, desafios e políticas. Cadernos de Prospecção, Salvador, v. 13, n. 2, p. 459-476, 2020.

HARAMBOURE, M. et al. Modelling the control of Aedes albopictus mosquitoes based on sterile males release techniques in a tropical environment. Ecological Modelling, [S.I.], (in press):109002. doi: 10.1016/j.ecolmodel.2020.109002, 2020.

HEAVENS, N. G.; WARD, D. S.; MAHOWALD, N. M. Studying and Projecting Climate Change with Earth System Models. Nature Education Knowledge, [S.I.], v. 4, n. 5, p. 4. 2013.

HIMICS, M.; LISTORTI, G.; TONINI, A. Simulated economic impacts in applied trade modelling: A comparison of tariff aggregation approaches. Economic Modelling, [S.1.], v. 87, p. 344-357, 2020. 
IBGE - INSTITUTO BRASILEIRO DE GEOGRAFIA E ESTATÍSTICA. Estimativas da População. [2020]. Disponível em: https:/www.ibge.gov.br/estatisticas/sociais/populacao/9103-estimativas-depopulacao.html? =\&t=resultados. Acesso em: 27 mar. 2020.

IHME - IHME COVID-19 health service utilization forecasting team. New IHME projection sees COVID-19 deaths in Brazil at more than 125,000. 2020a. Disponível em: http://www. healthdata.org/news-release/new-ihme-projection-sees-covid-19-deaths-brazil-more-125000. Acesso em: 30 maio 2020.

IHME - IHME COVID-19 health service utilization forecasting team. Forecasting the impact of the first wave of the COVID-19 pandemic on hospital demand and deaths for the USA and European Economic Area countries. medRxiv 21 April 2020b.

IHME - IHME COVID-19 health service utilization forecasting team. New Projection Sees COVID-19 Deaths in Brazil at Nearly 90,000. 2020c. Disponível em: http://www.healthdata.org/ news-release/new-projection-sees-covid-19-deaths-brazil-nearly-90000. Acesso em: 4 maio 2020.

KISSLER, S. M. et al. Projecting the transmission dynamics of SARS-CoV-2 through the postpandemic period. Science, [S.I.], v. 14, Abr, 2020. DOI: 10.1126/science.abb5793.

LI, R. et al. Substantial undocumented infection facilitates the rapid dissemination of novel coronavirus (SARS-CoV2). Science, [S.I.], eabb3221, 2020. DOI: 10.1126/science. abb3221pmid:32179701.

MICHAUD, J.; KATES, J.; LEVITT, L. COVID-19 Models: Can They Tell Us What We Want to Know? 2020. Disponível em: https://www.kff.org/coronavirus-policy-watch/covid-19-models/. Acesso em: 29 maio 2020.

MINISTÉRIO DA SAÚDE. (Brasil). Coronavírus COVID-19. 2020. Disponível em: https:// coronavirus.saude.gov.br/. Acesso em: 12 abr. 2020.

MOREIRA, L. M. Teatro de temática científica : conceituação, conflitos, papel pedagógico e contexto brasileiro. Ciência e Educação, [S.1.], v. 21, n. 2, p. 511-523, 2015.

OLIVEIRA, E. H. A. Coronavírus: prospecção científica e tecnológica dos fármacos em estudo para tratamento da Covid-19. Cadernos de Prospecção, Salvador, v. 13, n. 2, p. 412-423, 2020.

PEERI, N. C. et al. The SARS and MERS, and novel coronavirus (COVID-19) epidemics, the newest and biggest global health threats: what lessons have we learned? International Journal of Epidemiology, [S.1.], v. 22, Feb. 2020. DOI: 10.1093/ije/dyaa033.

PEREIRA, A. D. S. O processo de elaboração de peças de teatro científico na formação inicial de professores de química. Revista de la Facultad de Ciencia Y Tecnologia, [S.l.], v. 44, p. 185-200, 2018.

QUINTELLA, C. M. et al. Vacinas para Coronavírus (COVID-19; SARSCOV-2): mapeamento preliminar de artigos, patentes, testes clínicos e mercado. Cadernos de Prospecção, Salvador, v. 13, n. 1, 2020.

SARAIVA, I. Z.; OLIVEIRA, N. S. M. N.; MOREJON, C. F. M. Impactos das Políticas de Quarentena da Pandemia Covid-19, Sars-Cov-2, sobre a CT\&I Brasileira: prospectando cenários pós-crise epidêmica. Cadernos de Prospecção, Salvador, v. 13, n. 2, p. 378-396, 2020. 
SILVA, A. A. M. On the possibility of interrupting the coronavirus (COVID-19) epidemic based on the best available scientific evidence. Revista Brasileira de Epidemiologia, [S.l.], v. 23, e200021, 2020.

SOUSA JÚNIOR, J. H. et al. Da desinformação ao caos : uma análise das fake news frente à pandemia do Coronavírus (COVID-19) no Brasil. Cadernos de Prospecção, Salvador, v. 13, n. 2, p. 331-346, 2020.

SOUZA C. L. et al. COVID-19: O Brasil ainda pode conter uma catástrofe! 2020. Disponível em: http://COVID-19.ufsj.tech/. Acesso em: 10 abr. 2020.

TATUM, C. T. S. et al. Coronavírus no Processo de Impactação Científica Global. Cadernos de Prospecção, Salvador, v. 13, n. 2, p. 494-512, 2020.

VIGLIONE, G. Tens of thousands of scientists are redeploying to fight coronavirus. Nature. 2020. Disponível em: https://www.nature.com/articles/d41586-020-00905-9. Acesso em: 13 abr. 2020.

WALKER, P. G. T. et al. The Global Impact of COVID-19 and Strategies for Mitigation and Suppression. WHO Collaborating Centre for Infectious Disease Modelling, MRC Centre for Global Infectious Disease Analysis, Abdul Latif Jameel Institute for Disease and Emergency Analytics, Imperial College London. 2020.

WHO - WORLD HEALTH ORGANIZATION. Coronavirus disease 2019 (COVID-19) - Situation Report 76. 2020. Disponivel em: https://www.who.int/docs/default-source/coronaviruse/situationreports/20200405-sitrep-76-COVID-19.pdf?sfvrsn=6ecf0977_2. Acesso em: 9 abr. 2020.

WORLDOMETERS.INFO. COVID-19 Coronavirus Pandemic. Dover, Delaware, U.S.A. 2020. Disponível em: https://www.worldometers.info/coronavirus/. Acesso em: 13 abr. 2020,

WYNANTS L. et al Prediction models for diagnosis and prognosis of covid-19 infection: systematic review and critical appraisal. BMJ, [S.I.], v. 369, m1328, 2020.

\section{Sobre os Autores}

\section{Anderson Oliveira Latini}

E-mail: aolatini@ufsj.edu.br

Doutorado em Ecologia pela Unicamp em 2005.

Endereço profissional: Departamento de Ciências Exatas e Biológicas, Campus Sete Lagoas, Universidade Federal de São João del-Rei, Caixa Postal 56, Sete Lagoas, MG CEP: 35701-970.

\section{Celso Luiz Souza}

E-mail: celso.souza@ifsudestemg.edu.br

Doutorado em Modelagem Matemática Computacional pelo Centro Federal de Educação Tecnológica de Minas Gerais em 2018.

Endereço profissional: Instituto Federal do Sudeste de Minas Gerais, Campus São João del-Rei, Rua Américo Davim Filho, s/n, Vila São Paulo, São João del Rei, MG. CEP: 36301-358. 


\section{Daniela Chaves Resende}

E-mail: biologicaconsultoria@gmail.com

Doutorado em Entomologia pela UFV em 2005.

Endereço profissional: Departamento de Ciências Exatas e Biológicas, Campus Sete Lagoas, Universidade Federal de São João del-Rei, Caixa Postal 56, Sete Lagoas, MG. CEP: 35701-970.

\section{Carla Fabiana Gouvêa Lopes}

E-mail: carla.gouvea@if.sudestemg.gov.br

Graduação Tecnológica em Informática Empresarial pela Unipac em 2002.

Endereço profissional: Instituto Federal do Sudeste de Minas Gerais, Campus São João del-Rei, Rua Américo

Davim Filho, s/n, Vila São Paulo, São João del Rei, MG. CEP: 36301-358.

\section{Dárlinton Barbosa Feres Carvalho}

E-mail:darlinton@ufsj.edu.br

Doutorado em Informática pela PUC-Rio em 2013.

Endereço profissional: Departamento de Computação, Universidade Federal de São João del-Rei, Av. Visconde do Rio Preto, s/n, Prédio dos Cursos de Ciência da Computação e Zootecnia, $3^{\circ}$ andar, Sala 313, Colônia do Bengo, São João del Rei, MG. CEP: 36301-360. 